\title{
Utilization of Community Health Information System and Associated Factors in Health Posts of Hadiya Zone, Southern Ethiopia
}

\author{
Anheal Hintsa Zegeye \\ Hadiya zone health department, Hossana, Ethiopia \\ Nigusse Mekonnen Kara \\ Wolaita Sodo University, College of Health Science and Medicine, School of Public Health, Sodo, Ethiopia \\ Bealu Betebo Bachore \\ Hadiya zone health department, Hossana, Ethiopia \\ Bahailu Balcha Bachore \\ Wolaita Sodo University, College of Health Science and Medicine, School of Public Health, Sodo, Ethiopia
}

\begin{abstract}
Background: the regular use of reliable health information from a well-designed routine health information system is indispensable for ensuring and sustaining improvements in health system performance. However, In Ethiopia the prevailing practice in terms of effectively utilizing health information is not satisfactory and utilization of health information at the local level is still a challenge.

Objective: To determine utilization of Community Health information systems and associated factors in health posts of Hadiya zone, Ethiopia.

Method: Facility based cross sectional study design was conducted in Health post of Hadiya zone, from February 1 to 15/2018 using both quantitative and qualitative approach. The calculated sample 420 Health Extension workers found in 210 Health posts was allocated to each district proportionally based on their number of health posts. Then, Health posts were selected by simple random sampling method from each District, and health extension workers found in the selected Health posts was part of the study. Quantitative data was collected using structured questionnaire. Multivariable logistic regression was used to identify significantly associated factors. For qualitative study, health extension workers who are not participated in quantitative study were selected purposively based on their work experience.

Result: Overall, Good utilization of community health information was $41 \%$. Monthly Supportive supervision $(\mathrm{AOR}=1.72 ; \mathrm{CI}=1.02,2.91)$, written feedback $(\mathrm{AOR}=5.33 ; \mathrm{CI}=3.27,8.69)$ and Compiled and send additional parallel reports ( $\mathrm{AOR}=4.41 ; \mathrm{CI}=2.35-8.30$ ) were factors significantly associated with utilization of community health information system.

In qualitative study, the existing tools were difficult to use and understand because they prepared in English language, high burden of workload and no adequate work force at health post level were the main challenge in using CHIS.

Conclusion and Recommendation: Utilization of health information at health post level was found to be far below the national expectation. It needs major improvement in frequency of supportive supervision, sending of written feedback and improving integrated reporting channel system.
\end{abstract}

Keywords: Community health information systems, Health information, Ethiopia

DOI: $10.7176 / \mathrm{JMPB} / 63-03$

Publication date: January $31^{\text {st }} 2020$

\section{Background}

Information is the crux of overall building blocks of health systems. Strengthening and availability of information will enable health managers to utilize the same for better policy-making, planning, implementation and monitoring and evaluation of health programs(1).

A health information system (HIS) refers to any organized effort to systematically collect, maintain and disseminate data relevant to the performance of a health system or any of its component parts. As such, any health system has potentially many health applications functioning within it(2).

Ethiopia has undertaken an extensive reform and re-design of the health management information systems in 2007 to enhance the existing health management information system (HMIS) at federal, regional, zonal, District and health facility levels to produce reliable and timely information for planning management and efficient decision-making. The FMOH has designed and implemented community health information system (CHIS) as part of reformed HMIS(3). 
A Community Health Information System (CHIS) was introduced to capture basic health and health related information by Health Extension Workers at household and individual level. The CHIS collects data on basic demographic statistics and health service delivery and utilization based on the health extension package delivered through the Health Extension Program. CHIS has been designed to extend effective information management and decision making to the grassroots level (4).

The regular use of reliable information from a well-designed routine health information system (RHIS) is indispensable for ensuring and sustaining improvements in health system performance. Efforts have also been made to improve the culture of information use, in particular at the point of data collection. Although some improvements have been observed, ensuring data of sufficient quality and promoting an information-use culture at the point of collection remains critical and challenging $(4,5)$.

It is difficult to promote and maintain quality of primary, secondary, and tertiary health care without the availability and effective utilization of micro level medical information(6).

Routine Health Information system in developing countries lack well function information system. A study conducted in South Africa, shows that data were occasionally used to inform health education sessions run at the clinics and as a reflection of their work burden. Which shows as a culture of reporting rather than a culture of using the information(7).

A study in western Kenya, show that there were challenges to the health workers (lack of knowledge on data analysis and interpretation) mentioned as barriers to information utilization. In addition, there was a lack of timely use of information which may have led to haphazard planning and health interventions that were unrelated to household health needs. The information-seeking behavior and use of information was poor(8).

Most health care providers in developing countries equate information systems with filling endless registers with names and addresses of patients, compiling information on diseases every week or every month, and sending out reports without adequate feedback(9).

In Ethiopia, the prevailing practice in terms of effectively utilizing information is not satisfactory. Despite the intensive effort to improve the efficiency of information systems in the past few years, the utilization of information at the local level is still a challenge(6).

In our country, several studies show these problems briefly. A study done in Dire Dawa administration, regarding the use of health information for decision-making, $65.3 \%$ of the respondent reported they use information to make decision. Among them 46.2\% use the information for future reference, $42.3 \%$ use to observe trends of service delivery and $11.5 \%$ to pass reports for other subsidy health offices respectively. In General based on set criteria for Health information utilization rate at Health post level was found 39.3\% which is below the expected level, but cumulative health information utilization was $53.1 \%$.(10).

A study conducted in Jimma Zone, Oromia Regional State, revealed that based on the set criteria utilization of collected data/information at facility level were $26.7 \%, 31.3 \%$ and $36.0 \%$ for Health Posts, Health Centers and District Health Offices respectively. The cumulative utilization of collected data/ tried to change data into information/ cumulatively in study area were $32.9 \%$. which is very low than the expecting level(11).

These problems are also appreciated in SNNPR region. A study in Gamo Gofa Zone, on data management Knowledge, practice and associated factors of HEW show the overall data management knowledge were $58.2 \%$ among in HEWs which is low according to operational definition set for measurement of data management knowledge(13).

In SNNPR, Measure Evaluation project conducted performance of routine health information assessment in August 2011 \& February 2012 which showed that all administrative health units in SNNPR, have performance review teams; only $50 \%$ of them reviewed HMIS data in the past quarter and $25-45 \%$ of them showed evidence of HMIS based decision making which is very low from the expected level(14).

Information use is critical for better decision and crucial to improve the health status of the community at large. According to Hadiya Zone Health department, 2009 report Health information utilization and data quality are the major challenge for the health sector performance. Therefore, this study will be used as indication of current experience and show major influencing factors for utilization of Community health information system.

\section{Methods}

Facility based cross sectional study design was conducted approach in Hadiya Zone, approximately 200 kilometers southwest of Ethiopia's capital city, Addis Ababa

The study used both quantitative and qualitative data collection method from February 1-15/2018

All Functional Health posts found in Hadiya zone, which implements Community health information system during the study period, were included in the study and Health posts with no health extension workers or with only One-health extension workers during study period was excluded from the study.

The sample size was calculated using single population proportion formula at a $95 \%$ confidence level, $80 \%$ power of the test and $5 \%$ margin of error, $\mathrm{P}=45.8 \%$ (the overall health information utilization in East Gojjam Zone)(12)..Considering $\mathbf{1 0 \%}$ for non-response rate the total required sample size were $\mathbf{4 2 0}$ 
There are 10-district and 1-town administration with a total 308 HP in Hadiya zone. There are 659 HEWs in the zone. The calculated sample $420 \mathrm{HEWs}$ (Found in $210 \mathrm{HP}$ ) was allocated to each district proportionally based on their number of health posts. (Since, there are two HEW in each HP we expect 210 health post to meet our sample size). Then, Health posts was selected by simple random sampling method from each district, and health extension workers found in the selected 210 HPs was part of the study. (Since, two HEWs for one HPs is national standard).

The dependent variable was Utilization of Community health information system while the following factors were included in the model as independent variables: Socio-demographic factors: (Age, Marital status, Educational status and Salary) Behavioral Factor:(Data quality checking skill ,Attitude towardsHIS tasks,Confidence levels for HIS tasks ) Organizational Factor:(Resource availability ,Training ,Supervision ,Feedback, Functional PRT) Technical Factor: (procedures, standards and guidelines, Complexity of the Reporting form and Procedures, Data collection process, Data collection system or method)

The questionnaire was prepared in English, translated to Amharic language to make it understandable by the study participants and then was retranslated to English by another person to check whether the transition was consistent.

The questionnaire was adapted from the Performance of routine health information system management framework (PRISM) assessment tool (21). This questionnaire used to assess Organizational, behavioral and technical determinants. Organizational and technical factors were assessed by interviewing one of the HEW (particularly head of HEW) and both HEW will be participated for assessing behavioral and Socio-demographic factors.

To supplement the quantitative study In-depth interview was used.

Ten data collectors with diploma in health information technician (HIT) and two supervisors with degree in any health professionals who had experience in CHIs monitoring and had been involved in other similar field surveys was recruited.

Pretest was done on 5\% of the subjects on Kembata Tembaro zone, Kachabira District

First, data were checked manually for its completeness and consistency. Each completed questionnaire was assigned a unique code and entered to Epi-data version 3.1. Then, data were exported into SPSS version 20 for data processing and analysis. Frequencies, proportions, and summary statistics were used to describe the study population in relation to relevant variables and presented in tables. Bivariate analysis was carried out to identify variables that are significantly associated with utilization of community health information system.

Those Variables in bivariate analysis at $\mathrm{p}$-value less $<0.25$ were included in multivariate logistic regression then, multivariate logistic regression analysis was performed for those factors that showed a statistically significant association in bivariate analysis. Finally, variables whose $p$ value less than $0.05(p<0.05)$ in logistic regression were considered as statistically significant association. Qualitative study was the data was analyzed using thematic manual analysis.

\section{Results}

Among the total study participant $420 \mathrm{HEW}$ (from 210 Health posts) ,404 Health extension workers (found in 202 Health post) were included in the analysis, which make the response rate 96\%.Data for 16 HEW was incomplete, most of them have only One HEW during the study period.

\section{Characteristics of the study participants}

More than half $(52 \%)$ of the respondents were within the age of 26-30 years old with the mean age of 27.78 years with SD of 3.87 years and range from 20 to 46 years. Almost three-fourth $(76.5 \%)$ of the study participants was married. Among the total participant $70.8 \%$ were level III in educational status. Regarding on work experience of respondents, $44.6 \%$ of them had work experience of more than 10 years and $14.1 \%$ of them had work experience of less than 3 years. More than half $(54.2 \%)$ of the respondents had monthly salary ranging from 2001-3000 ETH.BIRR. (Table 1) 
Table 1. Socio-Demographic Characteristics of Health Extension Workers in Health post of Hadiya Zone, Southern Ethiopia, 2018

\begin{tabular}{|c|c|c|}
\hline Variables $n=404$ & Frequency(\#) & Frequency $(\%)$ \\
\hline \multicolumn{3}{|l|}{ Age (years) } \\
\hline$<25$ & 114 & 28.2 \\
\hline $26-30$ & 210 & 52 \\
\hline$>31$ & 80 & 19.8 \\
\hline \multicolumn{3}{|l|}{ Educational status } \\
\hline Level III & 286 & 70.8 \\
\hline Level IV & 118 & 29.2 \\
\hline \multicolumn{3}{|l|}{ Salary (ETH BIRR) } \\
\hline$<2000$ & 20 & 5.0 \\
\hline $2001-3000$ & 219 & 54.2 \\
\hline$>3001$ & 165 & 40.8 \\
\hline \multicolumn{3}{|l|}{ Marital status } \\
\hline Single & 95 & 23.5 \\
\hline Married & 309 & 76.5 \\
\hline \multicolumn{3}{|l|}{ Work Experience (years) } \\
\hline $1-3$ years & 57 & 14.1 \\
\hline 4-6 Years & 62 & 15.3 \\
\hline $7-9$ years & 105 & 26.0 \\
\hline$>10$ Years & 180 & 44.6 \\
\hline
\end{tabular}

\section{Organizational Factors}

Regarding to organizational factors according to their response, $45.0 \%$ of the participants had face shortage of CHIS material. Among this, $28.7 \%$ of them face shortage of Integrated $\mathrm{MCH}$ card, $15.3 \%$ of them face shortage of Family folder, and $25.7 \%$ of them had face shortage of monthly and quarterly reporting formats in the last six month. More than half (51\%) of the participants had received community health information trainings in the last 12 month. Only $35.6 \%$ of the participants were supervised every month in the last six month. One-fifth (22.8\%) of the participants were not supervised by near-by health centers, district health office or by other concerning bodies in the last six month. More than half (54.5\%) of the participants had received feedback from the next higher level. In terms of establishing PRT, only $71.3 \%$ of the participants had performance review team. Among the total participants, $38.1 \%$ were not discussed in health service performance or service utilization for the last six month with or without PRT.

In the qualitative study, Majority of the health extension workers described that properly utilization of CHIS tools, frequent supportive supervision from higher level and training are essential components for fully utilization of health information system. For example, one of the health extension workers who had 11 years of work experience said that;

\section{“...to utilize CHIS including cards, tally sheet and tickler boxes properly, training and close} supportive supervision is important."

\section{Technical Factors}

Regarding on availability of different reference material and manuals that help as a guide for health information utilization, among the total participants $32.2 \%$ were did not have manuals on health information utilization. More than one-fifth $(22.8 \%)$ of the participants compile and send parallel reports in addition to the standard of CHIS reports on weekly, monthly, quarterly and annual basis. The existing reporting formats were too complex or difficult to understand for $30.2 \%$ of the respondents. (Table 2 )

In qualitative study, majority of respondents had said that the existing report format is difficult to use and understand because it prepared in English language. For example, one-health extension workers who had 10 years of work experience state this;

"... Before this, we use report formats prepared in Amharic language, which is clear and easy to understand. However, CHIs report formats were difficult to use and understand. The main reason was it prepared in English language so it is not clear and difficult to understand"

Other health extension workers who had 12 years of work experience support this:

“...we always asked to our supporters from health centers to show us again and again because as it is prepared in English language it's difficult to understand due to language problem, even though supervisors from higher levels gave me explanation, we couldn't able to use it properly" 
Table 2: Technical factors in Health Post of Hadiya Zone, Southern Ethiopia, 2018

\begin{tabular}{|c|c|c|}
\hline Variables & Frequency (\#) & Frequency (\%) \\
\hline Standardized procedure/guideline on Health information utilization & 67.8 \\
\hline Yes & 274 & 32.2 \\
\hline No & 130 & 69.8 \\
\hline Report formats easy to use and understand & 282 & 30.2 \\
\hline Yes & 122 & 22.8 \\
\hline No & 92 & 77.2 \\
\hline Compile and send any other parallel report & 312 & \\
\hline Yes & & \\
\hline No &
\end{tabular}

\section{Behavioral Factors}

An individual behavioral factor was assessed through the ability/knowledge of HEW to undertake CHIS task, attitude of HEW workers towards the CHIS design, confidence level for CHIS task. Nearly three-fourth (73.8\%) of the participant performed data quality every month. Among those who were performing data quality, only $46.3 \%$ were used appropriate methods for data quality assurance (LQAS) technique. Surprisingly among the total respondent only $43.1 \%$ of the respondents know routine performance review meeting schedule. (Table 3 )

Table 3: Behavioral Factors of Health Extension Workers in Health Post of Hadiya Zone, Southern Ethiopia, 2018

\begin{tabular}{|c|c|c|}
\hline Variables & Frequency (\#) & Frequency $(\%)$ \\
\hline \multicolumn{3}{|l|}{ Performa Data quality } \\
\hline Yes & 298 & 73.8 \\
\hline No & 106 & 26.2 \\
\hline \multicolumn{3}{|l|}{ Method used for data quality } \\
\hline Use correct method (LQAS) & 187 & 46.3 \\
\hline Not Correct method & 111 & 27.5 \\
\hline Not conducted Data quality Check & 106 & 26.2 \\
\hline \multicolumn{3}{|l|}{ Frequency of PRT to take place } \\
\hline Don't know & 58 & 14.4 \\
\hline Quarterly & 62 & 15.3 \\
\hline Monthly & 174 & 43.1 \\
\hline weekly & 110 & 27.2 \\
\hline
\end{tabular}

\section{Attitude of Health extension workers for CHIs design}

Attitude of Health extension workers towards CHIs were measured using likert scale. In this study, when HEW whose score is greater than mean score was considered as having Favorable attitude. The mean value of health extension workers attitude towards design of CHIS was 43.25 (SD \pm 5.15 ). Almost half (47.0\%) of the health extension workers had Favorable attitude towards community health information design. Collecting information that is not used for decision-making discourages me, CHIS reporting forms are easy to fill and use and the existing community health information systems were easy to use and to understand were variables which showed lowest value.

Confidence level for CHIS tasks

Confidence level for CHIS tasks has seven components. The respondent were asked to rate their self-confidence for performing CHIS activities on a scale of zero to 100. Confidence level (Scale rating is from 0 to 100 , from no confidence to $100 \%$ confidence). For this study, average score of respondents equal or more than the mean score of confidence questions was considered as having "High confidence level". The mean value of health extension workers self-confidence toward CHIS activities was 61.25. Among the total respondents, 59.2\% of them had high self-confidence to perform CHIS tasks.

In qualitative study, majority of health extension workers states that the main challenge in using CHIS was high burden of workload and no adequate work force at health post level. Health extension workers are mostly busy working in dispersed rural villages, mainly through home visiting and they are spending time in collecting and reporting redundant data instead of serving the community.

For example, One Health extension workers said that;

“...Due to high burden of work load and geographical location of our kebele, I was too busy with field work and after that it's difficult for recording and collecting data, because I feel tired and sometimes I have forgotten to do that". 


\section{Utilization of community Health information}

Utilization of community health information focus on the ability to display and follow health indicators using table, graphs or other method, ability to calculate indicators for performance to compare with targets/eligible, to compare among type of services and to monitor data over time, ability to discuss on health facility performance, data quality, service utilization and other health services via performance review team (PRT), ability to made decisions based on discussion and ability to take action based on decisions. (Assessed by checking official minutes of performance review meetings maintained)

For this study, first all variables that indicates utilization of community health information were recode and after that one outcome variable was created. Then average score of respondents equal or more than the mean score was used to indicate as Good health information utilization and average score of respondents below the mean score was considered as poor utilization.

Based on this, Good utilization of the health information was $41.1 \%$. Only $67.1 \%, 63.9 \%$ and $57.4 \%$ of the study participant update and follow maternal, child, Hygiene and sanitation indicators respectively. Among the total participant, $61.9 \%$ compile and up-to-date population profiles. More than three-quarters $(80.7 \%)$ of the respondent calculate indicators performance from targets/Eligible, $63.9 \%$ of the respondents calculate and compare type of health services and $62.9 \%$ of respondents follow indicators those in need of monitoring over time. Almost half (49.0\%) of the participants had official minutes. Among those who have PRT and minutes $39.4 \%$ discuss on data quality, report timeliness/completeness and $47.6 \%$ discuss on health performance coverage, patient utilization, disease data, or medicine stock. Among those who made discussion, $43.1 \%$ of the participants made decisions and 34.2\% take action for decision. Only $31.2 \%$ of the participant had followed the action made during previous meeting. (Table 4)

Table 4: Utilization of community health information in Health posts of Hadiya Zone, Southern Ethiopia, 2018

\begin{tabular}{|c|c|c|}
\hline Variables & Frequency (\#) & Frequency $(\%)$ \\
\hline \multicolumn{3}{|c|}{ Display Maternal Health Indicators } \\
\hline Yes & 271 & 67.1 \\
\hline No & 133 & 32.9 \\
\hline \multicolumn{3}{|c|}{ Display Child health Indicators } \\
\hline Yes & 258 & 63.9 \\
\hline No & 146 & 36.1 \\
\hline \multicolumn{3}{|c|}{ Display Hygiene and sanitation Indicators } \\
\hline Yes & 232 & 57.4 \\
\hline No & 172 & 42.6 \\
\hline \multicolumn{3}{|c|}{ Compile and Up-to-date population profile } \\
\hline Yes & 250 & 61.9 \\
\hline No & 154 & 38.1 \\
\hline \multicolumn{3}{|c|}{ Calculate indicators performance from targets/Eligible } \\
\hline Yes & 326 & 80.7 \\
\hline No & 78 & 19.3 \\
\hline \multicolumn{3}{|c|}{ Comparisons among types of services coverage } \\
\hline Yes & 258 & 63.9 \\
\hline No & 146 & 36.1 \\
\hline \multicolumn{3}{|c|}{ Comparisons of data over time (Monitoring over time) } \\
\hline Yes & 254 & 62.9 \\
\hline No & 150 & 37.1 \\
\hline \multicolumn{3}{|l|}{ Routine PRT meetings } \\
\hline Yes & 288 & 71.3 \\
\hline No & 116 & 28.7 \\
\hline \multicolumn{3}{|c|}{ Official minutes of performance review meetings } \\
\hline Yes & 198 & 49 \\
\hline No & 206 & 51 \\
\hline \multicolumn{3}{|c|}{ Discussions on data quality, report timeliness and completeness } \\
\hline Yes & 159 & 39.4 \\
\hline No & 245 & 60.6 \\
\hline \multicolumn{3}{|c|}{ Discussions on health performance } \\
\hline Yes & 192 & 47.5 \\
\hline No & 212 & 52.5 \\
\hline
\end{tabular}




\begin{tabular}{|c|c|c|c|}
\hline \multicolumn{4}{|c|}{ made any decisions based on discussion } \\
\hline Yes & 174 & 43.1 & \\
\hline No & 230 & 56.9 & \\
\hline \multicolumn{4}{|l|}{ Any action taken place } \\
\hline Yes & 138 & 34.2 & \\
\hline No & 266 & 65.8 & \\
\hline \multicolumn{4}{|l|}{ Is there any Change } \\
\hline Yes & 136 & 33.7 & \\
\hline No & 268 & 66.3 & \\
\hline $\begin{array}{lcc}\text { Is } & \text { there } & \text { follow } \\
\text { made during the previous meetings }\end{array}$ & mechanism & the & action \\
\hline Yes & 126 & 31.2 & \\
\hline No & 278 & 68.8 & \\
\hline
\end{tabular}

\section{Factors associated with utilization of CHIS}

The main aim of this study is to assess utilization of CHIS and identify factors associated with CHIS utilization in health posts. First, bi-variable analysis was done for all independent variable with separately the outcome variables to identify candidate variables for multi-variable analysis. Variables with $\mathrm{p}<0.25$ during bi-variable analysis were selected as candidate for multi variable analysis. Based on this, Salary, Age, shortage of CHIs materials, supportive supervision, receiving feedback, sending of parallel report, Performing data quality and attitude towards CHIS task are variables selected for multi variable analysis. In addition, P-value less than 0.05 was taken as cut off point to label the significance of the variables in multivariable analysis. Accordingly one final model was developed.

Regular supportive supervision was one among the variable which has shown significant association $(\mathrm{P}<0.05)$ with utilization of community health information $(\mathrm{AOR}=1.72 ; \mathrm{CI}=1.02,2.91)$. Those health extension workers who receives monthly supportive supervision was 1.72 times more likely to utilize CHIS in good way compared to those who were not monthly supervised. Sending of written feedback also has significant relationship with utilization of community health information $(\mathrm{AOR}=5.33 ; \mathrm{CI}=3.27,8.69)$. Those who received feedback were 5.33 times more likely to utilized CHIs when compared with those who had not receiving feedback. Compiled and send other parallel report in addition to the standard of CHIS report on weekly, monthly, quarterly and annual basis also show significant association with utilization of community health information $(A O R=4.41: C I=2.35,8.30)$. Those who did not compile and send parallel report in addition to the standard report of CHIS were 4.41 times more likely to utilize of community health information compared to that are compiled and send parallel report in addition to the standard report of CHIS. (Table 5) 
Table 5: Factors associated with utilization of community health information in Health posts of Hadiya Zone, Southern Ethiopia, 2018

\begin{tabular}{|c|c|c|c|c|}
\hline \multirow[b]{2}{*}{ Variables } & \multicolumn{2}{|c|}{ Utilization of CHIS } & \multirow[b]{2}{*}{$\begin{array}{c}\text { COR } \\
95 \% \text { C.I. }\end{array}$} & \multirow[b]{2}{*}{$\begin{array}{l}\text { AOR } \\
95 \% \text { C.I. }\end{array}$} \\
\hline & Good utilization & Poor utilization & & \\
\hline \multicolumn{5}{|l|}{ Salary } \\
\hline$<2000$ ETH birr & $11(2.7 \%)$ & $9(2.2 \%)$ & $1.50(0.59-3.82)$ & $1.86(0.59-5.78)$ \\
\hline 2001-3000 ETH birr & $81(20 \%)$ & $138(34.2 \%)$ & $0.72(0.47-1.08)$ & $0.70(0.43-1.13)$ \\
\hline$>3001$ ETH Birr & $74(18.3 \%)$ & $91(22.5 \%)$ & 1 & 1 \\
\hline \multicolumn{5}{|l|}{ Age } \\
\hline$<25$ Years & $48(11.9 \%)$ & $66(16.3 \%)$ & $1.35(0.74-2.43)$ & $1.44(0.70-2.99)$ \\
\hline 26-30 Years & $90(22.3 \%)$ & $120(29.7 \%)$ & $1.39(0.81-2.37)$ & $1.45(0.78-2.69)$ \\
\hline$>31$ years & $28(6.9 \%)$ & $52(12.9 \%)$ & 1 & 1 \\
\hline \multicolumn{5}{|l|}{ Supportive supervision } \\
\hline No SS conducted & $33(8.2 \%)$ & $59(14.6 \%)$ & $0.98(0.57-1.66)$ & $1.09(0.59-2.00)$ \\
\hline Every Month & $72(17.8 \%)$ & $72(17.8 \%)$ & $1.75(1.11-2.76)$ & $1.72(1.02-2.91)^{*}$ \\
\hline More than a Month & $61(15.1 \%)$ & $107(26.5 \%)$ & 1 & 1 \\
\hline \multicolumn{5}{|l|}{ Feedback } \\
\hline Yes & $130(32.2 \%)$ & $90(22.3 \%)$ & $5.93(3.77-9.33)$ & $5.33(3.27-8.69)^{*}$ \\
\hline No & $36(8.9 \%)$ & $148(36.6 \%)$ & 1 & 1 \\
\hline \multicolumn{5}{|l|}{$\begin{array}{l}\text { Shortage of } \\
\text { materials }\end{array}$} \\
\hline Yes & $62(15.3 \%)$ & $120(29.7 \%)$ & $0.58(0.39-0.87)$ & $0.68(0.42-1.10)$ \\
\hline No & $104(25.7 \%)$ & $118(29.2 \%)$ & 1 & 1 \\
\hline \multicolumn{5}{|l|}{ Performing Data quality } \\
\hline Yes & $132(32.7 \%)$ & $166(41.1 \%)$ & $1.68(1.05-2.68)$ & $1.64(0.95-2.83)$ \\
\hline No & $34(8.4 \%)$ & $72(17.8 \%)$ & 1 & 1 \\
\hline \multicolumn{5}{|l|}{ Parallel report send } \\
\hline Yes & $19(4.7 \%)$ & $73(18.1 \%)$ & 1 & 1 \\
\hline No & $147(36.4 \%)$ & $165(40.8 \%)$ & $3.42(1.97-5.94)$ & $4.41(2.35-8.30)^{*}$ \\
\hline \multicolumn{5}{|l|}{ Attitude of HEW } \\
\hline Unfavorable Attitude & $81(20.0 \%)$ & $133(32.9 \%)$ & 1 & 1 \\
\hline Good Attitude & $85(21.0 \%)$ & $105(26.0 \%)$ & $1.32(0.89-1.97)$ & $1.38(0.87-2.20)$ \\
\hline
\end{tabular}

\section{Discussion}

Based on PRISM analytical framework of health information system performance, this study attempted to assess utilization of community health information and its associated factors in Health posts of Hadiya Zone, Southern Ethiopia. The overall Good utilization of community health information was $41.1 \%$ according to operational definition set for measurement of health information use. That is higher when compared with a studies in Harari region and Jimma Zone which was overall utilization of health information was 22.2 and $32.1 \%$ respectively $(11,24)$. The increment in this study is may be due to regular supportive supervision and written feedback down to the health post and the time of the study period. This study is lower when compared with a studies conducted in East Gojjam zone, Dire Dawa administration and Hadiya zone where the cumulative utilization of health information in the studies area was $45.8 \%, 53.1 \%$ and $69.3 \%$ respectively $(10,12,22)$. Since those studies were reported from health centers, the decrement in this study might be the lower attention paid by the government to health posts by providing supervision and feedback. However, in both studies the utilization of health information was below than the national expectation of information utilization that is $90 \%(6)$. In contrast, the level of utilization of community health information in our study was lower than a study conducted in Kenya, $69.9 \%$ of the community health workers use health information(25). This might be due to the difference in structure of health information system and health professional culture routine health information utilization.

Regular Supportive supervision is a key in addressing quality issues by helping to improve overall performance of HMIS especially for better achievement of information use. Among health extension workers who had regular supportive supervision was higher than those of their counter-parts were. The finding shows that those participants who were supervised monthly were 1.72 times more likely to have Good utilization than compared to those who were not monthly supervised. The result was supported by study conducted in Gamo Gofa zone and East Gojjam zone, those Health facility who were supervised regularly were 4 times and 2.6 more likely to have good knowledge and health information utilization respectively(12,13). This might be because of 
frequent supportive supervision has a significant role in identifying the gaps and improving health extension workers capacity. One of the most important mechanisms to improve health information utilization is regular supportive supervision. There for without regular supportive supervision it is difficult to improve information use culture at the point of data collection.

The essential role of feedback and external assistance in the form of supportive supervision is to reinforce behavior change. Dissemination of results for comparison of HI performance and peer review, when an HI's performance is critiqued and appreciated by those with similar responsibilities in another HI, are both powerful methods to induce and reinforce behavior change(17). This study also revealed the importance of written feedback. Those who had received feedback were 5.33 times more likely to utilize community health information when compared to those who had not received feedback. A Study conducted in Eastern Ethiopia and East Gojjam zone, shows that those health workers who had regular feedback had 2.19 and 2.2 times higher initiatives to utilize routine health information systems when compared to health workers who had no feedback respectively $(10,12)$. This might be due to those who receive regular feedback might receive fruitful and guidance to utilize health information. Reports were also showed that lack of feedback and promotion of data use by decision makers decreased staff motivation to seek, use or share data(20,26).

Compiled and sending of parallel reports in addition to the standard of CHIS report on weekly, monthly, quarterly and annual basis also show significant association with utilization of community health information. In our study, those participants who are not compile and send parallel reports were 4.41 times more likely to utilized health information system than those who compile and send Parallel report in addition to the standard of CHIS report. This might be due to lost their time by collecting different type of reports which create high burden of workload and Prohibited them from fully utilized for their own purpose rather than sending for higher level. An interpretative qualitative case study conducted in Amhara region, reported that health ex working at the health posts who were supposed to collect additional data that showed there is still a tendency to use multiple data collection tools and reports. Offering extra data collection and reporting tools for HEWs was create an additional burden to their regular work(27).

Reports also show that an integrated reporting channel that provides a single source for HMIS data is essential for an effective and for an efficient system (one that minimizes operational time and cost)(17). The Ethiopia HSTP strongly suggest that regularly assess reporting mechanism and implement interventions to ensure accountability towards "Zero Tolerance for Parallel Reporting" (6). Despite the main aim of HMIS/CHIS were standardization, integration and simplification of the health information system that includes integrating data from different programs into a shared channel from which all derive their information. However, this study indicates existing of complex and multi-channeled reporting tools and system and there is high data demand at higher level.

\section{Conclusion}

More than half of the health extension workers had poor routine health information utilization. Utilization of health information at health post level was found to be far below the national expectation that is aimed to achieved $90 \%$ of health information utilization(6). Organizational and technical factors were the main determinant of health information utilization. Frequent supportive supervision, written feedback, Compiled and sending of parallel report were factors, which have shown significant associations with routine health information utilization. It needs major improvement in frequency of supportive supervision, written feedback and follow up and improving integrated reporting channel system. All HP must take action after feedback received to improve health information utilization. Improve frequency of Integrated supportive supervision and program specific supervision. Develop simple and unified data collection tools and report formats based on Amharic language or local language. Revise number of Health extension workers needed based on workload. Further research is recommended for assessing culture of health information utilization at the lower level where data are generated using only qualitative approach

\section{Acknowledgments}

Our gratitude goes to supervisors, data collectors, study respondents and Hadiya Zone Health Department for facilitating the study.

\section{Authors' contributions}

Aneheal Hintsa : Involved from the conception and design, acquisition of data, analysis and interpretation, Niguse Mekonnen: Involved from the inception to design, acquisition of data, analysis and interpretation and drafting the manuscript, Bealu betebo : Involved in the analysis \& interpretation of data, Bahailu Balcha : Involved in interpretation and manuscript preparation. Finally all authors read and approved the final manuscript. 


\section{Competing interests}

The authors declare that they have no competing interests

Funding

This study has been sponsored by wolaita Sodo University

\section{Ethical issues}

The study was approved by Research Ethics Review Committee, college of health science and medicine, wolaita sodo university. The supportive letter was written from the college to SNNPR health bureau. Participants were informed clearly about the purpose, risk and benefit of the study and informed, voluntary, written and signed consent was obtained from them. The confidentiality of responses was maintained throughout the research process by giving code for participant. Personal privacy and cultural norms was respected.

The data will be available when requested

\section{References}

1. WHO regional office for S.Asia. Utilization of Health Information for Decision-Making 2008.

2. Vital wave Consulting. Health Information Systems in Developing Countries May 2009

3. FDREMo Health. National HMIS quality improvement mentorship guidelines December 2014.

4. EFMoHealth. Information Revolution Roadmap. April 2016.

5. Evaluation UM. Guidelines for Data Management Standards in Routine Health Information Systems. June 2014.

6. FDRoE MoHealth. Health sector transformation plan. 2015.

7. A Garrib NS, A McKenzie, L Dlamini, T Govender, J Rohde, K Herbst. An evaluation of the District Health Information System in rural South Africa. 2007.

8. Otieno Careena Flora1, Kaseje Margaret1, Kaseje Dan1 Perspectives on utilization of community based health information systems in Western Kenya 2012.

9. WHO Geneva. Design and implementation of health information systems 2000.

10. Kidist Teklegiorgis KT, Gebremeskel Mirutse, Wondwossen Terefe Factors associated with low level of health information utilization in resources limited setting, Eastern Ethiopia 2013.

11. Sultan Abajebel1 CJ, Waju Beyene2 Utilization of health information system at district level in jimma zone oromia regional state, south west Ethiopia. 2009.

12. Atsede Mazengia Shiferaw1* DTZ, Solomon Assefa1 and Melaku Kindie Yenit2. Routine health information system utilization and factors associated thereof among health workers at government health institutions in East Gojjam Zone, Northwest Ethiopia 2013.

13.Sewunet Sako Shagake1 MYMaAAZ. Data Management Knowledge, Practice and Associated Factors of Ethiopian Health Extension Workers in Gamo Gofa Zone, Southern Ethiopia:2012.

14. USAID HMIS scale up Ethiopia. HMIS Scale-Up in Ethiopia 2012.

15.WHO-MBHSS. Health information systems. 2010.

16. Pact IW, DC Field Guide for Data Quality Management 2014.

17. FMo Health. Health Management Information System (HMIS) Strategic Plan for Ethiopian Health Sector 2008.

18. FMoHealth Ethiopia. Community Health Information System Data Recording and Reporting 2011.

19. FDRoE Mo Health. POLICY AND PRACTICE INFORMATION FOR ACTION Better Information Better Decision Better Health. April 2014(Quarterly Health Bulletin).

20. Measure evaluation. Data Demand and Information Use in the Health Sector.

21.MEASURE Evaluation/JSI.Performance of Routine Information System Management (PRISM) Guide. June 30,2009

22. Ermias Abera1* KD, Taye Letta2 \& Desalegn Tsegaw3 Utilization of Health Management Information System and Associated Factors in Hadiya Zone Health Centers, Southern Ethiopia. 2014.

23. Evaluation M. Assessment of Health Management Information System (HMIS) Performance in SNNPR, Ethiopia 2013.

24. Mahtsentu M. Assessment of Health Management Information System in Harari Regional State June 2010.

25. WEKESA RN. Utilization of the health information management system by community health workers in the AMREF facility in kibera,Nairobi county, Kenya. December 2014.

26. Evaluation M. A Review of Constraints to Using Data for Decision Making August 2010.

27. Zufan Abera DAMTEWa aASM. From Multiple Register to Family folder: The Transition of Data Collection and Reporting Tools for Health Extension Workers in Ethiopia 2011. 